\title{
Retinal haemorrhage and fatal stroke in an infant with fibromuscular dysplasia
}

\author{
A D M Currie, C R Bentley, P A Bloom
}

\begin{abstract}
Non-accidental injury should be suspected and excluded in any infant found to have intracranial and retinal haemorrhage of unknown aetiology. This can be a sensitive issue for both medical staff and parents. We present a case in which the underlying cause of intracranial and retinal haemorrhage was fibromuscular dysplasia. It was a diagnosis made only at postmortem examination and it illustrates the diagnostic difficulty such cases may present.

(Arch Dis Child 2001;84:263-264)
\end{abstract}

Keywords: retinal haemorrhage; stroke; fibromuscular dysplasia; non-accidental injury

\section{Case report}

An 11 month old male infant presented with a six hour history of irritability, vomiting, pyrexia $\left(38^{\circ} \mathrm{C}\right)$, and hypotonia. He was hypertensive (blood pressure $180 / 110 \mathrm{~mm} \mathrm{Hg}$ ). It was thought he may have meningitis or encephalitis. Cefotaxime and acyclovir were administered and an intravenous infusion of dextrose saline was commenced. Twelve hours after presentation, his level of consciousness deteriorated (Glasgow Coma Scale 6). His pulse was 220 per minute, his blood pressure was

Western Eye Hospital, London NW1 5YE, UK A D M Currie

C R Bentley

P A Bloom

Correspondence to: Dr A D M Currie,

Department of

Ophthalmology, Queen's

Medical Centre, Nottingham NG7 2UH, UK

ali.currie4@virgin.net

Accepted 7 November 2000

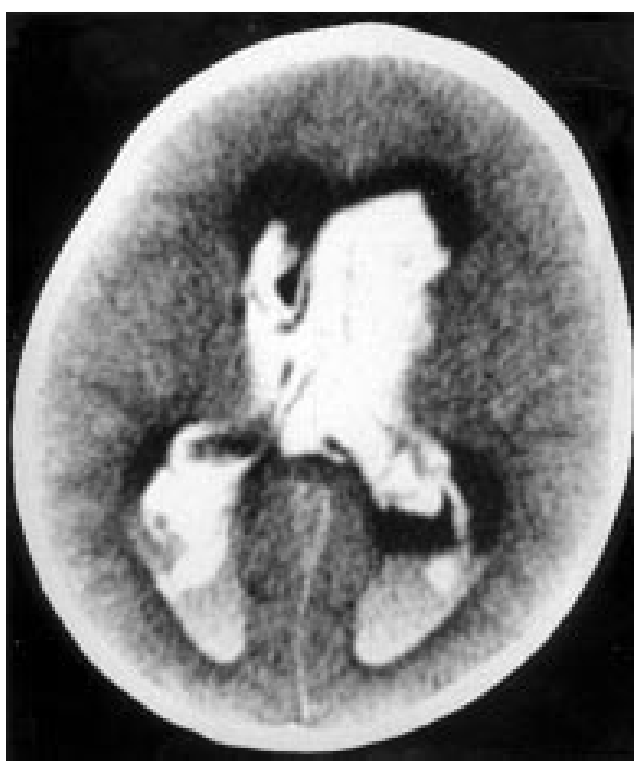

Figure 1 Computed tomography scan showing intracerebral and intraventricular haemorrhage. unrecordable, and his respiratory rate was 60 per minute. He was transferred to another hospital and admitted directly to the paediatric intensive care unit where he was intubated and ventilated. A brain computed tomography scan showed intracerebral and intraventricular haemorrhage with hydrocephalus (fig 1). Fundus examination revealed extensive subhyaloid and intraretinal haemorrhages (fig 2). Mannitol $20 \%$ was administered. Systemic steroids were commenced and a loading dose of phenytoin was given. Intravenous colloid and a dobutamine infusion maintained the blood pressure. It was questioned whether this presentation could be attributed to nonaccidental injury but there was no superficial evidence of injury to the child. In particular, there was no bruising, no subconjunctival haemorrhages, no lacerations, and the frenulum of the tongue was intact. A skeletal survey was normal with no evidence of skull fracture. There was no family history of vascular abnormalities, haematological conditions, or neurometabolic disorders. While on the intensive care unit, it became apparent that the raised intracranial pressure had led to significant brainstem dysfunction and the infant died three days later.

The postmortem report described marked concentric hypertrophy of the left ventricle and

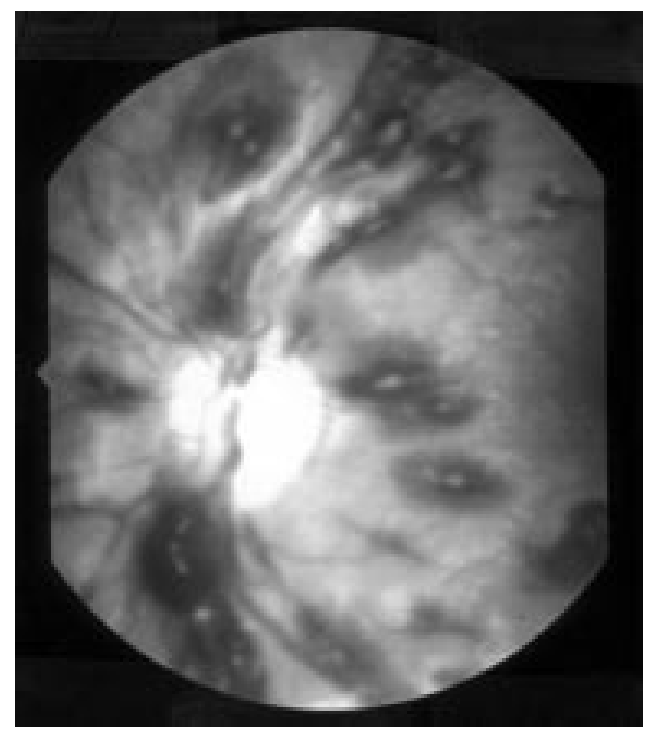

Figure 2 Fundus photograph showing extensive intraretinal haemorrhage. 
fibrinoid necrosis of small cerebral arterioles, in keeping with severe antemortem hypertension. Extreme intimal proliferation was noted in the aorta which was considered too severe to have arisen as a consequence of hypertension in the short period of this infant's life and indicated the presence of underlying fibromuscular dysplasia (FMD) of intimal type. This was considered to be the mechanism for the underlying hypertension, by causing renal ischaemia, as evidenced by focal scarring.

\section{Discussion}

Fibromuscular dysplasia is a nonatheromatous, non-inflammatory, multifocal segmental angiopathy of uncertain aetiology, ${ }^{1}$ although humoral, mechanical, and genetic factors may play a role. One autopsy series has shown its incidence in the general population to be approximately $1 \% .^{2}$ Its incidence in the paediatric population is not known exactly, but is rare. Symptoms depend on the vascular bed involved and the severity of the resulting stenosis. The histological classification is based on the predominant site of dysplasia in the arterial wall: the intima, media, or adventitia. Medial fibromuscular dysplasia is the most frequent type in the paediatric population. Histological examination revealed the FMD in this case to be of intimal type which accounts for only approximately $1-5 \%$ of all fibromuscular arterial lesions. ${ }^{3}$ This case is atypical in that it is unusual for FMD to present with clinical signs in such a young infant. The presentation of FMD is usually as ischaemic rather than haemorrhagic stroke. When intracerebral haemorrhage is observed it is more commonly as a late complication of established renovascular hypertension.

Intracranial and retinal haemorrhage can be presenting findings in haematological conditions, for example, idiopathic thrombocytopenic purpura (platelet count usually less than $10-15 \times 10^{9} / 1$ ), haemophilia, and other coagulopathies. Sepsis can also present in this way as can a subarachnoid haemorrhage caused by a ruptured intracranial aneurysm, an acute subdural haemorrhage caused by trauma, or a sudden rise in intracranial pressure secondary to a bleed from an arteriovenous malformation or cavernous haemangioma. A neurometabolic disorder, glutaric aciduria type 1 (GA1) is a rare autosomal recessive inborn error of metabolism which can present with coexisting intracranial and retinal haemorrhage. ${ }^{4}$

In the absence of an initially identifiable aetiology in this child, non-accidental injury was suspected. Retinal haemorrhages occur in $65-89 \%$ of infants who have sustained nonaccidental injury as a result of shaking injuries, ${ }^{5}$ the extent correlating with the severity of intracranial pathology. The head of the infant is particularly vulnerable to whiplash type injuries as sustained during shaking, caused by numerous factors. This includes the proportionately larger and relatively unsupported head, the pliability of the sutures and fontanelles that allow stretching of the calvarium, the greater deformability of the unmyelinated brain, and the greater percentage of cerebrospinal fluid as a proportion of total cerebral content. ${ }^{6}$ In a case of non-accidental injury one would therefore expect to see generalised brain contusion or bilateral subdural haematomas which may be induced secondary to the greater shearing stresses exerted on the intracranial blood vessels. Ocular examination did reveal widespread retinal haemorrhages similar to those seen in non-accidental injury, but none of the above described intracranial findings were shown in this case.

Basic coagulation studies and a platelet count should be performed in cases of coexisting intracerebral and retinal haemorrhage to exclude a bleeding disorder. Blood cultures should be performed if sepsis is suspected. In certain circumstances, urinary glutaric acid and glutarylcarnitine and plasma carnitine should be performed to exclude an inborn error of metabolism. Computed tomography scanning or magnetic resonance imaging with contrast should be performed, and conventional angiography may be required to exclude an arteriovenous malformation, cavernous haemangioma, aneurysm, or fibromuscular dysplasia. In the latter case angiography may show the classic "string of beads" stenoses. The importance of excluding the above mentioned diagnoses in genuine cases of non-accidental injury is legal; lawyers defending the perpetrators might make use of these rare conditions to cast doubt on their client's guilt. It would be helpful for the paediatrician to be able to provide evidence to exclude such diagnoses. A blood pressure reading performed in earlier life would have highlighted a problem in this infant. Routine infant blood pressure screening may have prevented this tragedy.

This case highlights the diagnostic challenge intracranial and retinal haemorrhage in infancy can present. Non-accidental injury is an important differential diagnosis, but is in part a diagnosis of exclusion, and other rare causes of retinal and intracranial haemorrhage must be ruled out.

1 Belen D, Bolay H, Firat M, et al. Intracranial fibromuscular dysplasia. Angiology 1996;47:627-32.

2 Heffelfinger MJ, Holley KE, Harrison EG Jr, Hunt JC. Arterial fibromuscular dysplasia studied at autopsy. $A m \dot{f}$ Clin Pathol 1970;54:274

3 Stanley JC, Gewertz BL, Bove EL, et al. Arterial fibrodysplasia: histopathologic character and current etiofibrodysplasia: histopathologic character and
logic concepts. Arch Surg 1975;110:561-6.

4 Hoffmann GF, Athanassopoulos S, et al. Clinical course, early diagnosis, treatment, and prevention of disease in glutaryl-CoA dehydrogenase deficiency. Neuropaediatrics 1996;27:115-23.

5 Billmire ME, Myers PA. Serious head injury in infants: accident or abuse? Pediatrics 1985;75:340-2.

6 Caffey J. On the theory and practice of shaking infants: its potential residual effects of permanent brain damage and mental retardation. Am f Dis Child 1972;124:161-9. 